$$
\begin{aligned}
& \text { Pontifícia Universidade C Cólica } \\
& \text { DO RIO DE JANEIRO }
\end{aligned}
$$

Hélio Nigri

\title{
Indústrias Criativas de Base Tecnológica: Estudo para o Desenvolvimento de um Cluster de Inovação no Brasil
}

\author{
Tese de Doutorado
}

Tese apresentada ao Programa de Pós-graduação em Engenharia de Produção do Departamento de Engenharia Industrial da PUC-Rio como parte dos requisitos parciais para obtenção do título de Doutor em Engenharia de Produção

Orientador: $\quad$ Prof. Paulo Roberto Tavares Dalcol Co-Orientador: Prof. Antonio José Junqueira Botelho

Rio de Janeiro

Setembro de 2009 


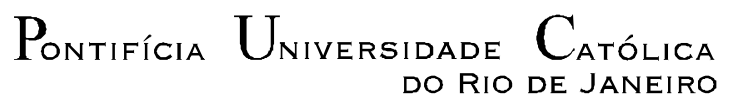

Hélio Nigri

\title{
Indústrias Criativas de Base Tecnológica: Estudo para o Desenvolvimento de um Cluster de Inovação no Brasil
}

\begin{abstract}
Tese apresentada como requisito parcial para obtenção do título de Doutor pelo Programa de Pós-Graduação em Engenharia de Produção da PUC-Rio. Aprovada pela Comissão Examinadora abaixo assinada.
\end{abstract}

Prof. Paulo Roberto Tavares Dalcol Orientador Departamento de Engenharia Industrial - PUC-Rio

Prof. Antônio José Junqueira Botelho Co-orientador

Programa de Pós-Graduação em Metrologia, Qualidade e Inovação

PUC-Rio

Prof. Bruno Feijó Departamento de Informática - PUC-Rio

Prof. Paulo Bastos Tigre UFRJ

Prof. Marcos do Couto Bezerra Cavalcanti COPPE/UFRJ

Prof. Alex da Silva Alves

Programa de Pós-Graduação em Metrologia, Qualidade e Inovação

PUC-Rio

Prof. José Eugenio Leal Coordenador Setorial do Centro Técnico Científico - PUC-Rio 
Todos os direitos reservados. É proibida a reprodução total ou parcial do trabalho sem autorização da universidade, do autor e do orientador.

\section{Hélio Nigri}

Graduado em Engenharia Civil pela Universidade Federal do Rio de Janeiro em 1983, Analista de Sistemas pela Pontifícia Universidade Católica do Rio de Janeiro (PUC-Rio) em 1985, Especialização em Administração pela PUC-Rio em 1987, Mestre em Informática pela PUC-Rio em 1991, desenvolveu pesquisa no Imperial College of Science, Tecnology and Medicine da University of London em 1996/1997. Foi professor dos Cursos de Análise de Sistemas e de Computação Gráfica Aplicada da Coordenadoria de Cursos de Extensão da PUC-Rio de 1989 a 1996. Trabalhou na Promon Engenharia de 1985 a 1993, sendo Gerente de Computação a última posição ocupada. É sócio-fundador da Nigraph Tecnologia, onde atua desde 1993.

Ficha Catalográfica

Nigri, Hélio

Indústrias criativas de base tecnológica : estudo para o desenvolvimento de um Cluster de Inovação no Brasil / Hélio Nigri ; orientador: Paulo Roberto Tavares Dalcol ; coorientador: Antonio José Junqueira Botelho. - 2009.

130 f. ; $30 \mathrm{~cm}$

Tese (Doutorado em Engenharia Industrial) - Pontifícia Universidade Católica do Rio de Janeiro, Rio de Janeiro, 2009.

Inclui bibliografia

1. Engenharia industrial - Teses. 2. Indústrias criativas de base tecnológica. 3. Clusters. 4. Inovação. 5. Ambiente criativo. 6. Megamídia. I. Dalcol, Paulo Roberto Tavares. II. Botelho, Antonio José Junqueira. III. Pontifícia Universidade Católica do Rio de Janeiro. Departamento de Engenharia Industrial. IV. Título. 
Esta tese é dedicada ao meu querido pai, meu grande incentivador, que deixou este mundo doze dias antes da defesa da tese.

Dedico também à minha esposa Karen e aos meus filhos Dan , Gabriel e Ruth. 


\section{Agradecimentos}

À minha esposa Karen pelo enorme incentivo, carinho, paciência e compreensão durante todo o doutorado e por ter segurado a barra sozinha durante os inúmeros fins-de-semana em que me dediquei ao doutorado em detrimento à minha família. Sem a sua ajuda e apoio eu não teria conseguido completar esta tese.

Aos meus queridos filhos Dan, Gabriel e Ruth que, na maneira particular de cada um, entenderam as minhas ausências em inúmeras programações infantis em função da tese. Tenho agora como meta recuperar o tempo que estive privado do convívio com eles.

Aos meus queridos pais, meus eternos agradecimentos pelo exemplo, carinho, apoio e incentivo irrestrito durante toda a minha vida.

Tive o privilégio de ter sido orientado pelos Professores Paulo Roberto Tavares Dalcol e Antonio José Junqueira Botelho. Nossas reuniões conjuntas discutindo conceitos e idéias foram momentos de intenso aprendizado para mim. Meus sinceros agradecimentos ao Professor Dalcol pela confiança, pelas sugestões valiosas e objetivas e por estar sempre pronto a ajudar no que fosse necessário. Ao Professor Botelho por ter me introduzido com brilhantismo nos temas sobre empreendedorismo e inovação, pela amizade e pelas inúmeras idéias e sugestões para a pesquisa durante todo o programa de doutorado.

Ao Professor Bruno Feijó do departamento de Informática da PUC-Rio, membro da banca tanto no doutorado quanto na minha defesa de mestrado no programa de Informática da PUC-Rio há 18 anos atrás. Tenho muito a agradecer ao Professor Bruno pelas mais de duas décadas de amizade e conselhos. Especificamente em relação ao Doutorado, agradeço por ter me incentivado a ingressar no programa em Engenharia de Produção, pela sugestão do tema de pesquisa e pelo interesse e participação ativa durante toda a pesquisa.

A José Alberto Aranha, Diretor do Instituto Genesis da PUC-Rio, pelo apoio e incentivo para ingressar no programa de doutorado. 
A Professora Maria Angela Campelo de Melo pelo apoio nos primeiros anos do doutorado.

Ao meu querido amigo Cláudio Wasserman, amigo de todas as horas, que além do apoio e incentivo nos momentos mais difíceis, ainda se dispôs a fazer a revisão gramatical do texto.

Aos meus companheiros da Nigraph Tecnologia pelas oportunidades de troca, discussões e vivências no desenvolvimento de produtos inovadores que, de alguma forma, influenciaram nos pontos de vista expostos neste trabalho.

Por último e mais importante de tudo, agradeço a D`us, fonte da vida, por ter me dado forças e removido obstáculos durante toda esta caminhada. 


\section{Resumo}

Nigri, Hélio; Dalcol, Paulo Roberto Tavares (Orientador); Co-Orientador: Botelho, Antonio José Junqueira (Co-Orientador). Indústrias Criativas de Base Tecnológica: Estudo para o Desenvolvimento de um Cluster de Inovação no Brasil. Rio de Janeiro, 2009. 130p. Tese de Doutorado Departamento de Engenharia Industrial, Pontifícia Universidade Católica do Rio de Janeiro.

Esta tese tem como objetivo estabelecer os fatores determinantes para o desenvolvimento de uma nova indústria - a indústria criativa de base tecnológica (ICBT) - no Brasil, tendo como foco a formação de clusters de inovação. A partir da análise da literatura sobre a emergência e a evolução de indústrias criativas e as novas oportunidades geradas pela convergência digital é apresentada uma definição e a cadeia produtiva das ICBTs, uma indústria com características singulares e um enorme potencial de crescimento. A crescente importância do lócus regional no processo de geração de inovações e do impacto da inovação para o desenvolvimento econômico, inclusive regional, põe em evidência o papel fundamental do estabelecimento de instituições como aprendizado coletivo, comunicação informal e criação de conhecimento, conexões com os líderes, indústria de capital de risco e ambientes criativos como elementos centrais de redes regionais de produção de inovações. A tese identifica e avalia os fatores determinantes de sucesso de clusters de inovação a partir da análise comparativa de dez clusters e parques tecnológicos do exterior e, em seguida, aprofunda a análise destes para os casos de Israel e Taiwan, os mais bem sucedidos da indústria de alta tecnologia mundial depois do Silicon Valley. A pesquisa examina na seqüência o Plano de Ação de Ciência, Tecnologia e Inovação do Governo Brasileiro sob o ponto de vista do estímulo ao desenvolvimento de ICBTs no país. Finalmente, são apresentadas as vantagens locacionais, estruturais e culturais de uma região, a Cidade do Rio de Janeiro, que apresenta um alto potencial para o desenvolvimento de um cluster de ICBTs no país. A partir dos resultados da avaliação dos fatores determinantes nos casos bem-sucedidos de clusters de inovação de alta tecnologia, da natureza e características singulares das ICBTs e das lacunas institucionais identificadas na política brasileira, é apresentado um conjunto de recomendações que visa transformar a Cidade do Rio de Janeiro em um centro de referência internacional da ICBT.

\section{Palavras-chave}

Indústrias criativas de base tecnológica, clusters, inovação, ambiente criativo, megamídia. 


\section{Abstract}

Nigri, Hélio; Dalcol, Paulo Roberto Tavares (Advisor); Botelho, Antonio José Junqueira (Co-Advisor). Technology Based Creative Industries: A Study for the Development of an Innovation Cluster in Brazil. Rio de Janeiro, 2009. 130p. Doctorate Thesis - Departamento de Engenharia Industrial, Pontifícia Universidade Católica do Rio de Janeiro.

This thesis aims to establish the determinant factors for the development of a new industry - the technology-based creative industries (TBCI) - in Brazil, focusing on the formation of innovation clusters. Building on the results of a literature review of the emergence and evolution of the creative industries and of the new opportunities generated by the digital convergence, the thesis presents a definition and the productive chain of the TBCI, a singular industry with an enormous growth potential. The growing importance of the regional locus in the innovation generation process and the impact of innovation on economic development highlight the fundamental role of establishing institutions such as collective learning, informal communication and knowledge creation, connection to leaders, the venture capital industry and the creative environment as central elements of innovation producing regional networks. The thesis identifies and assesses the determinants of success of innovation clusters based on a comparative analysis of ten clusters and science parks around the world and, then, makes an in-depth analysis of the Israel and Taiwan cases, two of the most successful clusters in the high technology industry, next to Silicon Valley. The research also examines the Brazil's Science, Technology and Innovation Action Plan, from the perspective of policies and mechanisms therein to promote the development of the TBCIs in the country. Finally, this work introduces the location, structural and cultural advantages of a region, the city of Rio de Janeiro, which holds a great potential to develop a TBCI cluster in Brazil. From the assessment of the determinant factors in the most successful high technology innovation clusters; the analysis of nature and singularities of the TBCIs and the identification of the institutional gaps in the Brazilian policy, it is presented a set of recommendations that aim to transform the City of Rio de Janeiro in an international reference of the TBCI.

\section{Keywords}

Technology based creative industries, clusters, innovation, creative environment, megamedia. 


\section{Sumário}

1. INTRODUÇÃO 15

1.1. Relevância do Tema $\quad 15$

1.2. Objetivos $\quad 19$

1.3. Metodologia 20

1.4. Estrutura da Tese 20

2. CLUSTERS, EMPREENDEDORISMO E O PAPEL DAS PMEs 26

2.1. Caracterização dos Aglomerados Produtivos Locais 26

2.2. Natureza e Evolução dos Clusters de Inovação 28

2.3. A Economia do Empreendedorismo 32

2.4. O Papel das PME`s na Nova Economia 34

2.5. Colaboração em Clusters de Inovação Tecnológica 35

3. ECONOMIA CRIATIVA 37

3.1. Indústrias Criativas $\quad 37$

$\begin{array}{ll}\text { 3.2. Classe Criativa } & 39\end{array}$

3.3. Cidades Criativas $\quad 42$

3.4. A Indústria Criativa no Brasil 44

4. INDÚSTRIAS CRIATIVAS DE BASE TECNOLÓGICA 47

4.1. Definição 47

4.2. Escopo e Abrangência $\quad 49$

4.3. Principais Características 53

4.4. A Cadeia Produtiva da ICBT 57

4.5. O Ambiente Criativo 60

5. A EXPERIÊNCIA INTERNACIONAL DE CLUSTERS E PARQUES TECNOLÓGICOS 62

5.1. Tipologias Existentes $\quad 62$

5.2. Tipologia Adotada $\quad 65$

5.3. Análise Comparativa de Clusters e Parques Tecnológicos 67

5.4. O Silicon Wadi de Israel 73

5.5. O Hsinchu Science Park de Taiwan 83

6. DESENVOLVIMENTO DE UM CLUSTER DE INDÚSTRIAS CRIATIVAS DE BASE TECNOLÓGICA NA CIDADE DO RIO DE JANEIRO

6.1. Estratégias do Governo Brasileiro para Ciência, Tecnologia e $\begin{array}{ll}\text { Inovação } & 89\end{array}$

6.2. A Situação Atual das ICBTs na Cidade do Rio de Janeiro 92

6.3. O Potencial de Crescimento das ICBTs na Cidade do Rio de Janeiro 95

6.4. O Projeto do VisionLab 98

6.5. Outras Propostas Existentes 99

6.6. O Ambiente Criativo da Cidade do Rio de Janeiro 101 
6.7. Caminhos para Formação de um Cluster de ICBTs na Cidade do Rio de Janeiro

7. CONSIDERAÇÕES FINAIS E CONCLUSÕES 110

7.1. Considerações Finais 110

7.2. Conclusões 119

7.3. Sugestões para Trabalhos Futuros 122

REFERÊNCIAS BIBLIOGRÁFICAS 123

ANEXO I: Distribuição dos Países em Função do Estágio de suas Economias 


\section{Lista de Figuras}

$\begin{array}{ll}\text { Figura } 1 \text { - Estrutura da Tese } & 21\end{array}$

Figura 2 - Indústrias Criativas de Base Tecnológica 48

Figura 3 - Exportação de Novas Mídias, 1996-2005 55

Figura 4 - Taxa de Crescimento das Exportações de Novas Mídias, 2000-2005

Figura 5 - Cadeia Produtiva das Indústrias Criativas de Base Tecnologia

Figura 6 - Subprocessos e as Atividades Principais dentro do Processo de Produção da Cadeia Produtiva das ICBTs

\section{Lista de Tabelas}

Tabela 1 - Rede de Cidades Criativas segundo a UNESCO

\section{Lista de Destaques}

Destaque 1 - Um típico representante da Classe Criativa

Destaque 3 - Carioca retorna do exterior para criar empresas de tecnologia no Rio 


\section{Lista de Abreviaturas e Siglas}

ABDI - Agência Brasileira de Desenvolvimento Industrial.

APL - Arranjo Produtivo Local.

C,T\&I - Ciência, Tecnologia e Inovação.

CAPES - Coordenação de Aperfeiçoamento de Pessoal de Nível Superior.

CNPq - Conselho Nacional de Desenvolvimento Científico e Tecnológico.

EAD - Educação a Distância.

ERSO - Eletronics Research and Services Organizations, Taiwan.

FAPERJ - Fundação Carlos Chagas Filho de Amparo à Pesquisa do Estado do

Rio de Janeiro.

FINEP - Financiadora de Estudos e Projetos.

ICBT - Indústria Criativa de Base Tecnológica.

ICT - Instituição de Ciência e Tecnologia.

INPI - Instituto Nacional de Propriedade Industrial.

ITRI - Industrial Technology Research Institute, Taiwan.

LCD- Liquid Crystal Display.

MCT - Ministério de Ciência e Tecnologia.

P\&D - Pesquisa e Desenvolvimento.

P,D\&I - Pesquisa, Desenvolvimento e Inovação.

PITCE - Política Industrial, Tecnológica e de Comércio Exterior do Governo Brasileiro.

PUC-Rio - Pontifícia Universidade Católica do Rio de Janeiro.

PME - Pequena e Média Empresa.

RBV - Rede Brasileira de Visualização. 
RedeSist - Rede de Pesquisa em Sistemas Produtivos e Inovativos Locais do Instituto de Economia da UFRJ.

SEBRAE - Serviço de Apoio às Micro e Pequenas Empresas.

SPIL - Sistemas Produtivos e Inovativos Locais.

TI - Tecnologia da Informação.

TIC - Tecnologia da Informação e Comunicação.

UFRJ - Universidade Federal do Rio de Janeiro.

UNCTAD - United Nations Conference on Trade and Development.

UNESCO - United Nations Educational, Scientific and Cultural Organization. 
“Hilel dizia: Se eu não for por mim, quem será por mim?

Mas se eu for só por mim, o que sou eu?

E se não for agora, quando?”

Código de Leis Judaico - A Ética dos Pais, Capítulo I, parágrafo 14. 